\title{
Interference Control for Cognitive Network with High Mobility
}

\author{
Yuanxuan Li, Gang Zhu, Siyu Lin, Ke Guan, and Bo Ai \\ State Key Laboratory of Rail Traffic Control and Safety, Beijing Jiaotong University, Beijing 100044, China \\ Correspondence should be addressed to Yuanxuan Li; g.liyuanxuan@gmail.com
}

Received 19 April 2013; Revised 9 July 2013; Accepted 23 July 2013

Academic Editor: Chih-Hao Lin

Copyright (C) 2013 Yuanxuan Li et al. This is an open access article distributed under the Creative Commons Attribution License, which permits unrestricted use, distribution, and reproduction in any medium, provided the original work is properly cited.

\begin{abstract}
Interference control (IC) between the secondary system and the primary system is an important issue for underlay cognitive radio network (CRN). The secondary system should limit the interference power to primary system by adjusting its transmission power. Many relevant works have been done based on the assumption of the quasistatic channel which is not suitable for the fast timevarying fading channel; the performance of IC in underlay CRN will become worse when the channel varies fast. This paper studies the IC issue in high mobility environment. By considering the channel state information (CSI) outdatedness, a short frame structure scheme and a mean interference power constraint scheme are proposed to reduce the influence of CSI outdatedness on IC performance. Furthermore, by considering the channel estimation error, a spherical error region model based robust IC scheme is designed as well. The proposed IC schemes of the secondary system are converted to the power allocation problems, and then they are formulated to optimization problem whose objects are to maximize the capacity of the secondary system with the interference constraints. The above optimization problems are solved by the water-filling style method. The simulation results show that the proposed IC schemes can effectively control the interference power to the primary system.
\end{abstract}

\section{Introduction}

Traditionally, the different spectrum bands have been allocated to different mobile communication systems, such as Wideband Code Division Multiple Access (WCDMA) system, Long-Term Evolution (LTE) system, and LongTerm Evolution Advanced (LTE-A) system, by the spectrum authorization. The fixed spectrum allocation policy is the most convenient way for spectrum management avoiding the interference among different communication systems. However, the current spectrum management policies have seriously limited the development of mobile communication systems [1]. Cognitive network technology has been considered as a promising way to improve the spectrum efficiency. In cognitive radio networks (CRNs), the users are divided into two classes: primary users (PUs) and secondary users (SUs). There are two operation types of CRNs: underlay and interweave (overlay) CRNs. For interweave CRNs, the SUs can use the spectrum band only if the PUs are inactive in this band [2]. If the PUs are under heavy-load status over long term, the SUs can hardly use the spectrum bands. To meet the increasing quality of service (QoS) requirements of
SUs, the underlay CRN has been proposed, which permits the SUs to utilize the spectrum whenever the PUs are active or idle [3]. In this case, SUs can get more freedom and better spectrum utilization. However, to guarantee the PUs' QoS, the SUs should control their transmission power to avoid interfering with the PUs.

The underlay CRNs require that the interference from SUs does not disturb the signal detection and decoding of PUs, so the interference power should be limited under a predefined threshold at primary receiver (PR). The interference control (IC) plays a key role on the CRNs operation. How to maximize the capacity of the secondary system with interference constraint is an open issue for the CRNs optimization. In [4-7], there were several power allocation schemes with IC constraints in underlay CRNs. The design of interference constraints for SUs has been proposed in [8]. The distribution of interference power from SU has been discussed in [9]. In [10], Rabbachin et al. presented a statistical IC model considering the influence of channel fading. However, most IC schemes assumed that the wireless channel is quasistatic, which cannot fully reflect the nature of fading channel. The IC model with quasistatic channel assumption especially in the 
high mobility environment [11] will became invalid over time. Recently, some researchers started to concern the impact of the mobile fading channel for IC at SUs. Using the channel correlation coefficient to model the channel variation, Qiu et al. designed the interference constraints for the transmission schemes of SUs [12]. The time variation property of channel fading brings more challenges for IC in the secondary system. For the SU, the IC design should consider the impact of time-varying channel in different movement speed situations. Furthermore, since the IC constraints design relies on the channel state information (CSI), the channel estimation error will lead to the failure of IC. Therefore, the CSI outdatedness and channel estimation error should be considered for IC in the high mobility environment.

This paper focuses on the design of IC schemes for the secondary system in the high mobility environment. The IC schemes of the secondary system are converted to the power allocation problems. These problems can be formulated to optimization problem whose objects are to maximize the capacity of the secondary system with the interference constraints. The contributions are fourfold. First, in order to mitigate the influence of the CSI outdatedness, a short frame structure for IC scheme in normal mobility environment is proposed. The power allocation results are given by waterfilling style solutions. Second, for high mobility environment, the IC schemes with mean interference power constraint are proposed; then the power allocation problem is transformed to a convex problem by Jensen's inequality. Third, in order to cope with the channel estimation error problem, the robust IC scheme based on spherical error region model is proposed as well. Similar to the second IC scheme, the solution can be given through solving the approximated convex problem. Finally, the above three IC schemes are evaluated in different environments. Compared with the existing schemes, the simulation results show that the proposed IC schemes can control the interference power to the primary system effectively.

The rest of the paper is organized as follows: Section 2 describes the underlay system model. Section 3 proposes three interference control schemes. The secondary user power allocation schemes for IC will be analyzed in detail. The simulation results of interference control with mobile environment are presented in Section 4. The conclusions are made in Section 5.

\section{System Model}

The system model of underlay CRN is shown in Figure 1. The primary network has the primary base station (PBS) and the primary user (PU) which are interfered by the secondary base station (SBS) and the SU. The SU and the SBS should limit their transmission power to meet the requirement of signal to interference plus noise ratio (SINR) at the PU and the PBS. The CSIs between the SU(SBS) and the PBS(PU) are denoted by $H_{I_{\mathrm{UL}}}$ and $H_{I_{\mathrm{DL}}}$. The $H_{s_{\mathrm{UL}}}$ and $H_{s_{\mathrm{DL}}}$ represent uplink and downlink CSIs of the secondary system. The secondary devices (SU and SBS) are assumed to be able to control their transmission power by interference constraint using the $H_{I_{\mathrm{UL}}}$ and $H_{I_{\mathrm{DL}}}$. Both primary system

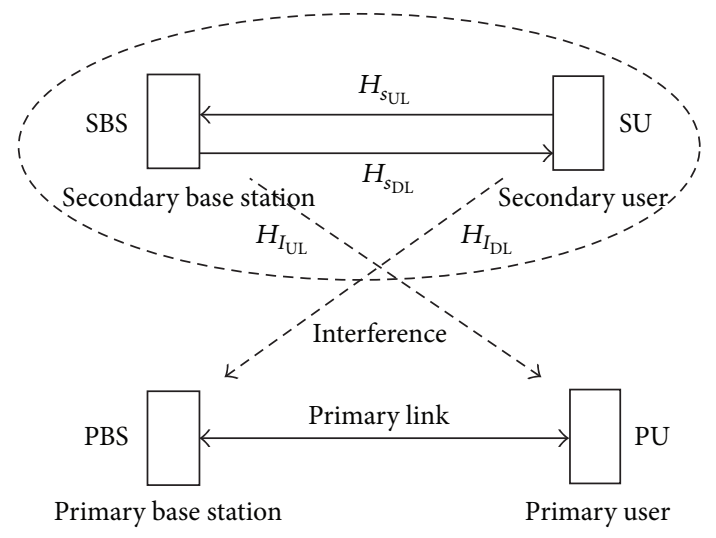

FIgURE 1: The underlay CR network topology.

and secondary system are orthogonal frequency division multiplexing (OFDM) systems with time division duplexing (TDD) and well synchronization characters. When the PU uploads the information to the PBS in the uplink, the SU transmits its signal to the SBS in the uplink at the same time slot. Similar to the uplink, the downlink at the secondary system is synchronized with the downlink at the primary system. The SUs access the CRN by the carrier sense multiple access with the collision avoidance (CSMA/CA) method which prevents the collisions between SUs. Assuming that the SU is moving (e.g., SU in a vehicle or a high speed train) and the PU is in low mobility status, the time-varying channel fading follows a zero-mean complex Gaussian process. The channel state of the primary network is quasistatic, which means that the channel state keeps steady in a frame duration. Secondary system utilizes the CSI $\left(H_{I_{\mathrm{UL}}}\right.$ and $\left.H_{I_{\mathrm{DL}}}\right)$ to design its IC scheme to control the interference. However, between the primary system and the secondary system, the channel state varies from symbol to symbol. Therefore, the CSI will be outdated in a frame duration. The relationship between $H(t, f)$ and $H(t+\tau, f)$ can be described as Clark-Jakes' model whose autocorrelation function is denoted by $\rho(\tau)=$ $\sigma_{0}^{2} J_{0}\left(2 \pi f_{d} \tau\right)$, where $\sigma_{0}^{2}$ is the variance of the channel, $J_{0}(\cdot)$ denotes the zero-order Bessel function of the first kind, $f_{d}$ is the maximum doppler frequency, and $\tau$ is the outdated time [13].

The channel capacity of SU at the $t$ th symbol can be calculated as

$$
C(t)=\sum_{f=1}^{M} B \log _{2}\left(1+\frac{P_{s}(t, f)\left|H_{s}(t, f)\right|^{2}}{N_{0}+I_{p}}\right),
$$

where $B$ is the bandwidth of the secondary system, $I_{p}$ is the interference power from the primary system, $N_{0}$ is the noise power, $P_{s}(t, f)$ is the transmission power of the secondary system at the $f$ th subband in the $t$ th symbol time, and $H_{s}(t, f)$ is the CSI of secondary link. The total transmission power constraint is $\sum_{f=1}^{M} P_{s}(t, f) \leq \Phi$, where $P_{s}(t, f) \geq 0$, for all $f$. The secondary system should control its transmission power with interference constraints while maximizing the channel capacity $C(t)$. The influence of CSI outdatedness is different in various movement speeds. 


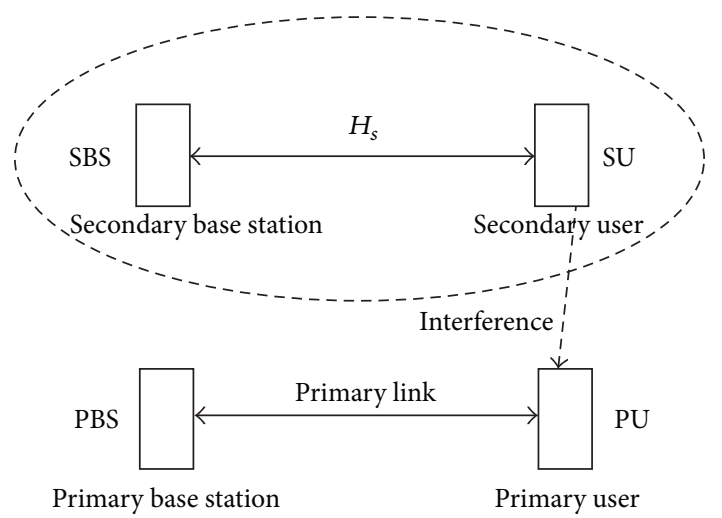

FIgURE 2: An underlay CRN with the primary system and the secondary system.

Furthermore, the channel estimation error cannot be avoided in time-varying fading channel, so it may lead to the failure of IC. Hence, how to control the interference in mobile environment is an open issue for the secondary system. In the following, several interference constraints are proposed to limit the interference power to the primary system. First, for the influence of CSI outdatedness, two IC schemes (a short frame structure in normal mobility environment and a mean interference power constraint) are designed in high mobility environment. Second, to cope with the channel estimation error, the channel estimation error on the basis of the spherical error region model for IC is formulated.

\section{The Power Allocations at Secondary System with Interference Constraints}

3.1. Traditional Interference Control Scheme. As shown in Figure 2, the existing works assume that the channel state keeps steady in one frame duration which does not vary from symbol to symbol. The $H_{I}\left(-\tau_{I}, f\right)$ represents the CSI between the SU and the PU which is delayed by $\tau_{I}$ symbols. The $H_{s}\left(-\tau_{s}, f\right)$ represents the CSI with the secondary system which is delayed by $\tau_{s}$ symbols.

The interference constraint in the $f$ th subband at the $t$ th symbol time is

$$
I(t, f)=P_{s}(t, f)\left|H_{I}\left(-\tau_{I}, f\right)\right|^{2} \leq \phi, \quad \forall f,
$$

where $\phi$ is the interference power threshold, which is the maximum interference power that the primary system can tolerate. The object of the optimization is maximizing the channel capacity of the secondary system. Therefore, the optimization problem with the interference power constraints can be formulated as

$$
\min \left\{-C(t)=\sum_{f=1}^{M} B \log _{2}\left(1+\frac{P_{s}(t, f)\left|H_{s}\left(-\tau_{s}, f\right)\right|^{2}}{N_{0}+I_{p}}\right)\right\},
$$

$$
\begin{gathered}
\text { s.t. } \quad \sum_{f=1}^{M} P_{s}(t, f) \leq \Phi, \quad-P_{s}(t, f) \leq 0, \\
P_{s}(t, f)\left|H_{I}\left(-\tau_{I}, f\right)\right|^{2} \leq \phi .
\end{gathered}
$$

Because it is a convex optimization problem, the Lagrangian formulation is given by

$$
\begin{aligned}
L\left(P_{s}(t, f)\right)= & -\sum_{f=1}^{M} B \log _{2}\left(1+\frac{P_{s}(t, f)\left|H_{s}\left(-\tau_{s}, f\right)\right|^{2}}{N_{0}+I_{p}}\right) \\
& +\sum_{f=1}^{M} \alpha\left(-P_{s}(t, f)\right) \\
& +\sum_{f=1}^{M} \beta\left(P_{s}(t, f)-\frac{\phi}{\left|H_{I}\left(-\tau_{I}, f\right)\right|^{2}}\right) \\
& +\gamma\left(\sum_{f=1}^{M} P_{s}(t, f)-\Phi\right),
\end{aligned}
$$

where $\alpha, \beta$, and $\gamma$ are Lagrangian multipliers. The KarushKuhn-Tucker (KKT) conditions are used to solve this optimization problem [12]

$$
\begin{gathered}
\frac{\partial L\left(P_{s}(t, f)\right)}{P_{s}} \\
=-\frac{B}{\ln 2} \frac{\left|H_{s}\left(-\tau_{s}, f\right)\right|^{2}}{N_{0}+I_{p}+\left|H_{s}\left(-\tau_{s}, f\right)\right|^{2} P_{s}(t, f)}-\alpha+\beta+\gamma \\
=0, \quad \alpha \geq 0, \quad \alpha P_{s}(t, f)=0, \\
\beta \geq 0, \quad \beta\left(P_{s}-\frac{\phi}{\left|H_{I}\left(-\tau_{I}, f\right)\right|^{2}}\right)=0 .
\end{gathered}
$$

The water-filling style solution is

$$
P_{s}(t, f)= \begin{cases}P_{A 1}, & \frac{B}{\mu \ln 2} \geq P_{A 1}+P_{A 2}, \\ \frac{B}{\mu \ln 2}-P_{A 2}, & P_{A 2}<\frac{B}{\mu \ln 2}<P_{A 1}+P_{A 2}, \\ 0, & \frac{B}{\mu \ln 2} \leq P_{A 2},\end{cases}
$$

where $P_{A 1}=\phi /\left|H_{I}\left(-\tau_{I}, f\right)\right|^{2}, P_{A 2}=\left(N_{0}+I_{p}\right) /\left|H_{s}\left(-\tau_{s}, f\right)\right|^{2}$, and $\mu$ is the Lagrange multiplier.

The constant constraint with the assumption of the quasistatic channel is a simplified way to control the interference power to the primary system. The actual interference power in the $f$ th subband at the $\tau$ th symbol is

$$
I(t, f)=P_{s}(t, f)\left|H_{I}(t+\tau, f)\right|^{2},
$$




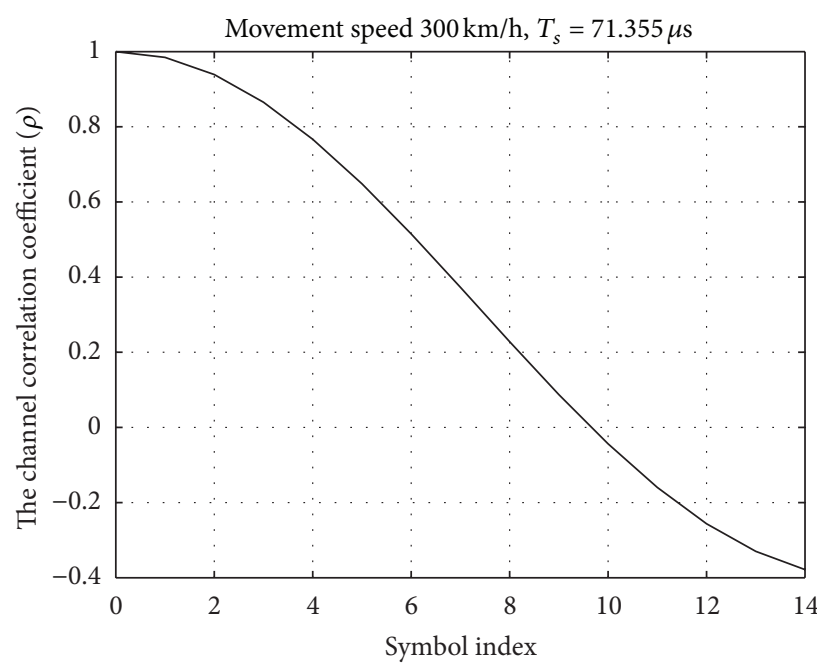

(a) The change of channel correlation

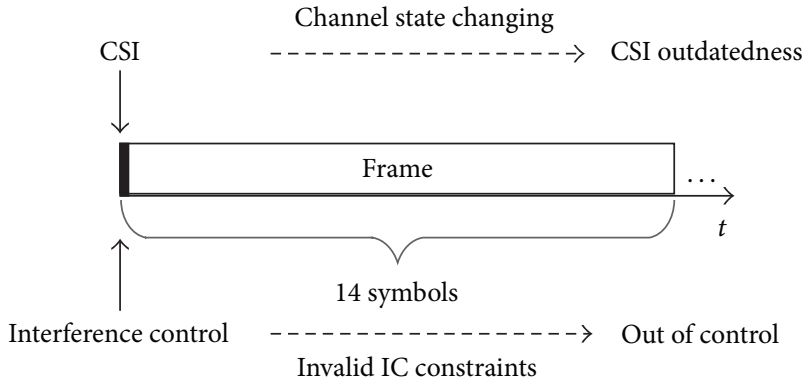

(b) The CSI outdatedness process

FIgURE 3: The CSI outdatedness in standard LTE transmission frame.

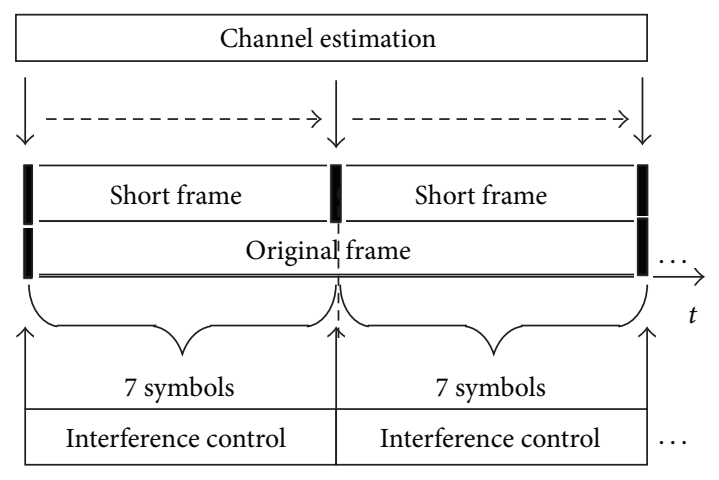

FIGURE 4: The short frame structure.

where $\tau$ is the number of outdated symbols. Taking the LTE system configuration, for example, there are 14 symbols in one frame. As shown in Figure 3, the CSI outdatedness process can be explained by the change of the channel correlation. The channel correlation coefficient $\rho$ decreases from symbol to symbol, which means that the gap between CSI and channel gain of current symbol becomes larger. The IC schemes with the quasistatic assumption cannot track the variation of fading channel, and, therefore, it will be invalid over time. This transmission scheme can only ensure the interference power under the predefined threshold in the beginning of a frame duration. In the rest of the time, the interference power may exceed the predefined threshold caused by CSI outdatedness. Thus, the CSI outdatedness should be considered in the high mobility environment.

3.2. Interference Control with Short Frame Structure. Only considering the interference between $\mathrm{SU}$ and $\mathrm{PU}$ is not proper for IC in underlay CRNs. As shown in Figure 1, the secondary system needs to control the interference power to $\mathrm{PU}$ and PBS. Both the primary system and the secondary system are the TDD systems with well synchronization. For the primary system, the SU disturbs the PBS in uplink, and the SBS disturbs the PU in downlink. For the channel between primary devices and secondary devices, $H_{I_{\mathrm{UL}}}\left(-\tau_{1}, f\right)$ and $H_{I_{\mathrm{DL}}}\left(-\tau_{2}, f\right)$ denote that the CSI knowledge is outdated by $\tau_{1}$ and $\tau_{2}$ symbols at subband $f$ in uplink and downlink, respectively. For the channel between SU and SBS, $H_{s_{\mathrm{UL}}}\left(-\tau_{s_{1}}, f\right)$ denotes that the CSI of uplink channel is outdated by $\tau_{s_{1}}$ symbols at subband $f$, and $H_{s_{\mathrm{DI}}}\left(-\tau_{s_{2}}, f\right)$ denotes that the CSI of downlink channel is outdated by $\tau_{s_{2}}$ symbols at subband $f$.

The CSI in the high mobility environment will be changed faster, which leads to more serious CSI outdatedness in time-varying fading channel. The time-varying fading channel varies in a small range; in a short time interval, its dynamic characteristics can be described by Clark-Jakes' model. Therefore, a new frame structure is required to fit the mobile environment. So, intuitively, the shorter frame duration means the smaller outdated time with CSI. In Figure 4, a short frame structure is proposed for IC in mobile environment which has 7-symbol duration. Shorting the frame length can improve the performance of IC and reduce the interference power from $\mathrm{SU}$

$$
\begin{aligned}
\min \{-C(t) & \\
\quad & \left.=\sum_{f=1}^{M} B \log _{2}\left(1+\frac{P_{s_{U L}}(t, f)\left|H_{S_{\mathrm{UL}}}\left(-\tau_{s_{1}}, f\right)\right|^{2}}{N_{0}+I_{p}}\right)\right\} \\
+ & \min \{-C(t) \\
& \left.=\sum_{f=1}^{M} B \log _{2}\left(1+\frac{P_{s_{\mathrm{DL}}}(t, f)\left|H_{s_{\mathrm{DL}}}\left(-\tau_{s_{2}}, f\right)\right|^{2}}{N_{0}+I_{p}}\right)\right\}
\end{aligned}
$$


s.t. $\quad P_{s_{\mathrm{UL}}}(t, f)\left|H_{I_{\mathrm{UL}}}\left(-\tau_{1}, f\right)\right|^{2} \leq \phi$,

$P_{s_{\mathrm{DL}}}(t, f)\left|H_{I_{\mathrm{DL}}}\left(-\tau_{2}, f\right)\right|^{2} \leq \phi$,

$\sum_{f=1}^{M} P_{s_{\mathrm{UL}}}(t, f) \leq \Phi, \quad-P_{s_{\mathrm{UL}}}(t, f) \leq 0$,

$\sum_{f=1}^{M} P_{s_{\mathrm{DL}}}(t, f) \leq \Phi, \quad-P_{s_{\mathrm{DL}}}(t, f) \leq 0$.

The solution is

$$
\begin{aligned}
& P_{s_{\mathrm{UL}}}= \begin{cases}P_{B 1}, & \frac{B}{\mu \ln 2} \geq P_{B 1}+P_{B 2}, \\
\frac{B}{\mu \ln 2}-P_{B 2}, & P_{B 2}<\frac{B}{\mu \ln 2}<P_{B 1}+P_{B 2}, \\
0, & \frac{B}{\mu \ln 2} \leq P_{B 2},\end{cases} \\
& P_{s_{\mathrm{DL}}}= \begin{cases}P_{B 3}, & \frac{B}{\mu \ln 2} \geq P_{B 3}+P_{B 4}, \\
\frac{B}{\mu \ln 2}-P_{B 4}, & P_{B 4}<\frac{B}{\mu \ln 2}<P_{B 3}+P_{B 4}, \\
0, & \frac{B}{\mu \ln 2} \leq P_{B 4},\end{cases}
\end{aligned}
$$

where $P_{B 1}=\phi /\left|H_{I_{\mathrm{UL}}}\left(-\tau_{1}, f\right)\right|^{2}, P_{B 3}=\phi /\left|H_{I_{D L}}\left(-\tau_{2}, f\right)\right|^{2}, P_{B 2}$ $=\left(N_{0}+I_{p}\right) /\left|H_{s}\left(-\tau_{s_{1}}, f\right)\right|^{2}$, and $P_{B 4}=\left(N_{0}+I_{p}\right) /\left|H_{s}\left(-\tau_{s_{2}}, f\right)\right|^{2}$.

3.3. Interference Control with Mean Interference Power Constraint. Considering high speed environments, such as high speed railway system, it is much harder to control the interference power $I(t, f)$ under a predefined threshold all the time due to the fast time-varying nature of the channel. Therefore, a mean interference power constraint based interference control scheme is proposed to maximize the mean capacity of the channel of the secondary system. $\phi$ is the interference threshold, and $I_{p}$ is the interference power from the primary system. The uplink and the downlink correlation coefficients between the SU to the PBS and the SBS to the PU are denoted by $\rho_{I_{\mathrm{UL}}}(t)=J_{0}\left(2 \pi f_{d}\left(t+\tau_{1}\right)\right)$ and $\rho_{I_{\mathrm{DL}}}(t)=J_{0}\left(2 \pi f_{d}\left(t+\tau_{2}\right)\right)$. And the $\rho_{s_{\mathrm{UL}}}(t)=J_{0}\left(2 \pi f_{d}\left(t+\tau_{s_{1}}\right)\right)$ and $\rho_{s_{\mathrm{DL}}}(t)=J_{0}\left(2 \pi f_{d}(t+\right.$ $\left.\tau_{s_{2}}\right)$ ) denote the uplink and downlink channel correlation coefficients between the SU and the SBS. Assuming that the uplink and the downlink channels are independent rayleigh channel, the CSI can be denoted as $\left|H_{I_{U L}}(t)\right|=\mid \mu_{U 1}(t)+$ $j \mu_{U 2}(t) \mid$ and $\left|H_{I_{\mathrm{DL}}}(t)\right|=\left|\mu_{D 1}(t)+j \mu_{D 2}(t)\right|$, which can be described as a complex Gaussian random processes and the variance is $2 \sigma^{2}$ [13]. The interference constraints of the uplink and the downlink can be described as

$$
\begin{aligned}
& E\left[\left.I(t, f)\right|_{H_{I_{\mathrm{UL}}}}\right]=P_{s_{\mathrm{UL}}}(t, f) E\left[\left|H_{I_{\mathrm{UL}}}(t, f)\right|^{2}\right] \leq \phi, \\
& E\left[\left.I(t, f)\right|_{\left.H_{I_{\mathrm{DL}}}\right]}\right]=P_{s_{\mathrm{DL}}}(t, f) E\left[\left|H_{I_{\mathrm{DL}}}(t, f)\right|^{2}\right] \leq \phi .
\end{aligned}
$$

For time-varying fading channel, the CSI can be expressed as

$$
\begin{aligned}
& H_{s_{\mathrm{UL}}}(t, f)=\rho_{s_{\mathrm{UL}}}(t) H_{s_{\mathrm{UL}}}\left(-\tau_{s_{1}}, f\right)+\widetilde{H}_{s_{\mathrm{UL}}}(t, f), \\
& H_{s_{\mathrm{DL}}}(t, f)=\rho_{s_{\mathrm{DL}}}(t) H_{s_{\mathrm{DL}}}\left(-\tau_{s_{2}}, f\right)+\widetilde{H}_{s_{\mathrm{DL}}}(t, f), \\
& H_{I_{\mathrm{UL}}}(t, f)=\rho_{I_{\mathrm{UL}}}(t) H_{I_{\mathrm{UL}}}\left(-\tau_{1}, f\right)+\widetilde{H}_{I_{\mathrm{UL}}}(t, f), \\
& H_{I_{\mathrm{DL}}}(t, f)=\rho_{I_{\mathrm{DL}}}(t) H_{I_{\mathrm{DL}}}\left(-\tau_{2}, f\right)+\widetilde{H}_{I_{\mathrm{DL}}}(t, f),
\end{aligned}
$$

where $\widetilde{H}_{I_{\mathrm{UL}}} \sim \mathcal{N}\left(0,1-\rho_{I_{\mathrm{UL}}}^{2}(t)\right), \widetilde{H}_{I_{\mathrm{DL}}} \sim \mathcal{N}\left(0,1-\rho_{I_{\mathrm{DL}}}^{2}(t)\right), \widetilde{H}_{s_{\mathrm{UL}}} \sim$ $\mathcal{N}\left(0,1-\rho_{s_{\mathrm{UL}}}^{2}(t)\right)$, and $\widetilde{H}_{s_{\mathrm{DL}}} \sim \mathcal{N}\left(0,1-\rho_{s_{\mathrm{DL}}}^{2}(t)\right)$.

Then, we obtain the expected value of CSI

$$
\begin{aligned}
& E\left[\left|H_{s_{\mathrm{UL}}}\right|^{2}\right]=\rho_{I_{\mathrm{UL}}}^{2}(t)\left|H_{s}\left(-\tau_{s_{1}}, f\right)\right|^{2}+\left(1-\rho_{s_{\mathrm{UL}}}^{2}(t)\right) 2 \sigma^{2}, \\
& E\left[\left|H_{s_{\mathrm{DL}}}\right|^{2}\right]=\rho_{I_{\mathrm{DL}}}^{2}(t)\left|H_{s}\left(-\tau_{s_{2}}, f\right)\right|^{2}+\left(1-\rho_{s_{\mathrm{DL}}}^{2}(t)\right) 2 \sigma^{2}, \\
& E\left[\left|H_{I_{\mathrm{UL}}}\right|^{2}\right]=\rho_{I_{\mathrm{UL}}}^{2}(t)\left|H_{I}\left(-\tau_{1}, f\right)\right|^{2}+\left(1-\rho_{I_{\mathrm{UL}}}^{2}(t)\right) 2 \sigma^{2}, \\
& E\left[\left|H_{I_{\mathrm{DL}}}\right|^{2}\right]=\rho_{I_{\mathrm{DL}}}^{2}(t)\left|H_{I}\left(-\tau_{2}, f\right)\right|^{2}+\left(1-\rho_{I_{\mathrm{DL}}}^{2}(t)\right) 2 \sigma^{2} .
\end{aligned}
$$

By substituting (13) and (14) into (8), power allocation with interference constraint can be written as

$$
\begin{aligned}
& P_{s_{\mathrm{UL}}} \leq \frac{\phi}{\rho_{I_{\mathrm{UL}}}^{2}(t)\left|H_{I}\left(-\tau_{1}, f\right)\right|^{2}+\left(1-\rho_{I_{\mathrm{UL}}}^{2}(t)\right) 2 \sigma^{2}}, \\
& P_{s_{\mathrm{DL}}} \leq \frac{\phi}{\rho_{I_{\mathrm{DL}}}^{2}(t)\left|H_{I}\left(-\tau_{2}, f\right)\right|^{2}+\left(1-\rho_{I_{\mathrm{DL}}}^{2}(t)\right) 2 \sigma^{2}} .
\end{aligned}
$$

Because the instantaneous channel capacity of the secondary system is changed over time, in the long run, the optimization object is the expected value of channel capacity which can be formulated as

$$
C=E\left[C_{\mathrm{UL}}+C_{\mathrm{DL}}\right]
$$

The optimization formulation at the $t$ th symbol is

$$
\begin{aligned}
& \min \left\{-E\left[\sum_{f=1}^{M} B \log _{2}\left(1+\frac{P_{s_{\mathrm{UL}}}(t, f)\left|H_{s_{\mathrm{UL}}}\left(-\tau_{s_{1}}, f\right)\right|^{2}}{N_{0}+I_{p}}\right)\right]\right\} \\
& \quad+\min \left\{-E\left[\sum_{f=1}^{M} B \log _{2}\left(1+\frac{P_{s_{\mathrm{DL}}}(t, f)\left|H_{s_{\mathrm{DL}}}\left(-\tau_{s_{2}}, f\right)\right|^{2}}{N_{0}+I_{p}}\right)\right]\right\}
\end{aligned}
$$




$$
\begin{array}{ll}
\text { s.t. } & P_{s_{\mathrm{UL}}} \leq \frac{\phi}{\rho_{I_{\mathrm{UL}}}^{2}(t) H_{I}\left(-\tau_{1}, f\right)+\left(1-\rho_{I_{\mathrm{UL}}}^{2}(t)\right) 2 \sigma^{2}}, \\
& P_{s_{\mathrm{DL}}} \leq \frac{\phi}{\rho_{I_{\mathrm{DL}}}^{2}(t) H_{I}\left(-\tau_{2}, f\right)+\left(1-\rho_{I_{\mathrm{DL}}}^{2}(t)\right) 2 \sigma^{2}}, \\
& \sum_{f=1}^{M} P_{s_{\mathrm{UL}}}(t, f) \leq \Phi, \quad-P_{s_{\mathrm{UL}}}(t, f) \leq 0 \\
& \sum_{f=1}^{M} P_{s_{\mathrm{DL}}}(t, f) \leq \Phi, \quad-P_{s_{\mathrm{DL}}}(t, f) \leq 0 .
\end{array}
$$

Unfortunately, the optimization function $E[-\log (\cdot)]$ is nonconvex. Because the $-\log (\cdot)$ is a convex function, there has $E[-\log (\cdot)] \geq-\log (E[\cdot])$ according to Jensen's inequality. Then the approximate function can be obtained $E[-\log (\cdot)] \approx$ $-\log (E[\cdot])$. The approximate channel capacity of the secondary system is

$$
\begin{aligned}
& E\left[C_{\mathrm{UL}}+C_{\mathrm{DL}}\right] \\
& \approx \min \left\{-\sum_{f=1}^{M} B \log _{2}\left(1+\frac{P_{s_{\mathrm{UL}}}(t, f) E\left[\left|H_{s_{\mathrm{UL}}}\left(-\tau_{s_{1}}, f\right)\right|^{2}\right]}{N_{0}+I_{p}}\right)\right\}, \\
& +\min \left\{-\sum_{f=1}^{M} B \log _{2}\left(1+\frac{P_{s_{\mathrm{DL}}}(t, f) E\left[\left|H_{s_{\mathrm{DL}}}\left(-\tau_{s_{2}}, f\right)\right|^{2}\right]}{N_{0}+I_{p}}\right)\right\},
\end{aligned}
$$

s.t. $\quad P_{s_{\mathrm{UL}}} \leq \frac{\phi}{\rho_{I_{\mathrm{UL}}}^{2}(t) H_{I}\left(-\tau_{1}, f\right)+\left(1-\rho_{I_{\mathrm{UL}}}^{2}(t)\right) 2 \sigma^{2}}$,

$$
\begin{aligned}
& P_{s_{\mathrm{DL}}} \leq \frac{\phi}{\rho_{I_{\mathrm{DL}}}^{2}(t) H_{I}\left(-\tau_{2}, f\right)+\left(1-\rho_{I_{\mathrm{DL}}}^{2}(t)\right) 2 \sigma^{2}}, \\
& \sum_{f=1}^{M} P_{s_{\mathrm{UL}}}(t, f) \leq \Phi, \quad-P_{s_{\mathrm{UL}}}(t, f) \leq 0, \\
& \sum_{f=1}^{M} P_{s_{\mathrm{DL}}}(t, f) \leq \Phi, \quad-P_{s_{\mathrm{DL}}}(t, f) \leq 0 .
\end{aligned}
$$

The solutions are

$$
P_{s_{\mathrm{UL}}}= \begin{cases}P_{\mathrm{C} 1}, & \frac{B}{\mu \ln 2} \geq P_{\mathrm{C} 1}+P_{\mathrm{C} 2}, \\ \frac{B}{\mu \ln 2}-P_{\mathrm{C} 2}, & P_{\mathrm{C} 2}<\frac{B}{\mu \ln 2}<P_{C 1}+P_{C 2}, \\ 0, & \frac{B}{\mu \ln 2} \leq P_{C 2},\end{cases}
$$

$$
P_{s_{\mathrm{DL}}}= \begin{cases}P_{\mathrm{C} 3}, & \frac{B}{\mu \ln 2} \geq P_{\mathrm{C} 3}+P_{\mathrm{C} 4}, \\ \frac{B}{\mu \ln 2}-P_{\mathrm{C} 4}, & P_{\mathrm{C} 4}<\frac{B}{\mu \ln 2}<P_{\mathrm{C} 3}+P_{\mathrm{C} 4}, \\ 0, & \frac{B}{\mu \ln 2} \leq P_{\mathrm{C} 4},\end{cases}
$$

where $P_{\mathrm{C} 1}=\phi /\left(\rho_{I_{\mathrm{UL}}}^{2}(t) H_{I}\left(-\tau_{1}, f\right)+\left(1-\rho_{I_{\mathrm{UL}}}^{2}(t)\right) 2 \sigma^{2}\right), P_{\mathrm{C} 2}=$ $\left(N_{0}+I_{p}\right) /\left(\rho_{s_{\mathrm{UL}}}^{2}(t) H_{s}\left(-\tau_{s_{1}}, f\right)+\left(1-\rho_{s_{\mathrm{UL}}}^{2}(t)\right) 2 \sigma^{2}\right), P_{\mathrm{C} 3}=$ $\phi /\left(\rho_{I_{\mathrm{DL}}}^{2}(t) H_{I}\left(-\tau_{2}, f\right)+\left(1-\rho_{I_{\mathrm{DL}}}^{2}(t)\right) 2 \sigma^{2}\right)$, and $P_{\mathrm{C} 4}=\left(N_{0}+\right.$ $\left.I_{p}\right) /\left(\rho_{s_{\mathrm{DL}}}^{2}(t) H_{s}\left(-\tau_{s_{2}}, f\right)+\left(1-\rho_{s_{\mathrm{DL}}}^{2}(t)\right) 2 \sigma^{2}\right)$.

3.4. Interference Control with the Channel Estimation Error. Since the channel estimation error of time-varying channel cannot be neglected, the estimated CSI between the secondary system and the primary system is imperfect. If the transmission power allocation scheme for the secondary system relies on the imperfect channel estimation result, the interference constraint will be

$$
I(t, f)=P_{s}(t, f)\left|\widehat{H}_{I}(t, f)\right|^{2}<\phi
$$

Then, the optimized transmission power of the secondary system $\left(P_{s}^{*}\right)$ with the channel estimation error may lead to the interference problem for primary system. The variance value of channel estimation error is $\sigma_{e}^{2}$.

The actual interference power is

$$
I(t, f)=P_{s}^{*}(t, f)\left\{\left|\widehat{H}_{I}(t, f)\right|^{2}+\sigma_{e}^{2}\right\}
$$

The interference power may exceed the interference threshold with the imperfect channel estimation result.

The estimation error is modeled as the spherical region $\left\|e_{I}\right\|<\sigma_{e}$, where the $\sigma_{e}$ is the maximum radius of error bound [14]. The channel model is described by $H_{I_{\mathrm{UL}}}(t, f)=\widehat{H}_{I_{\mathrm{UL}}}+$ $e_{I_{\mathrm{UL}}}$ and $H_{I_{\mathrm{DL}}}(t, f)=\widehat{H}_{I_{\mathrm{DL}}}+e_{I_{\mathrm{DL}}}$, where $\widehat{H}_{I_{\mathrm{UL}}}$ and $\widehat{H}_{I_{\mathrm{DL}}}$ are the estimated values of uplink and downlink channels, and $e_{I_{\mathrm{UL}}}$ and $e_{I_{\mathrm{DL}}}$ are the channel estimation errors. The variance value of the channel estimation error in uplink is assumed to be equal to the variance value of downlink channel estimation error, which can be uniformly denoted by $\sigma_{e}^{2}$.

The mean interference power constraint with imperfect CSI knowledge can be expressed as

$$
\begin{aligned}
E\left[\left.I(t, f)\right|_{\left.H_{I_{\mathrm{UL}}}\right]}\right. & =P_{s}(t, f) E\left[\left|H_{I_{\mathrm{UL}}}(t, f)\right|^{2}\right] \\
& =P_{s}(t, f) E\left[\left|\widehat{H}_{I_{\mathrm{UL}}}(t, f)\right|^{2}+\sigma_{e}^{2}\right] \\
& =P_{s}(t, f)\left\{\rho_{I_{\mathrm{UL}}}^{2}(t)\left|\widehat{H}_{I_{\mathrm{UL}}}\left(-\tau_{1}, f\right)\right|^{2}\right. \\
& \left.+\left(1-\rho_{I_{\mathrm{UL}}}^{2}(t)\right) 2 \sigma^{2}+\sigma_{e}^{2}\right\}
\end{aligned}
$$

$\leq \phi$, 


$$
\begin{aligned}
E\left[\left.I(t, f)\right|_{\left.H_{I_{\mathrm{DL}}}\right]}\right]=P_{s}(t, f)\left\{\rho_{I_{\mathrm{DL}}}^{2}(t)\left|\widehat{H}_{I_{\mathrm{DL}}}\left(-\tau_{2}, f\right)\right|^{2}\right. & \\
& \left.+\left(1-\rho_{I_{\mathrm{DL}}}^{2}(t)\right) 2 \sigma^{2}+\sigma_{e}^{2}\right\} \leq \phi .
\end{aligned}
$$

The mean capacity of the SU can be described as

$$
\begin{aligned}
& E\left\{C_{\mathrm{UL}}(t)+C_{\mathrm{DL}}(t)\right\} \\
& \approx \min \left\{-B \sum_{f=1}^{M} \log _{2}\left(1+\frac{P_{s_{\mathrm{UL}}}(t, f) E\left[\left|\widehat{H}_{s_{\mathrm{UL}}}\left(-\tau_{s_{1}}, f\right)\right|^{2}\right]}{N_{0}+I_{p}}\right)\right\}, \\
& \quad+\min \left\{-B \sum_{f=1}^{M} \log _{2}\left(1+\frac{P_{s_{\mathrm{DL}}}(t, f) E\left[\left|\widehat{H}_{s_{\mathrm{DL}}}\left(-\tau_{s_{2}}, f\right)\right|^{2}\right]}{N_{0}+I_{p}}\right)\right\},
\end{aligned}
$$

$$
\begin{array}{ll}
\text { s.t. } & P_{s_{\mathrm{UL}}}\left\{\rho_{I_{\mathrm{UL}}}^{2}(t)\left|\widehat{H}_{I_{\mathrm{UL}}}\left(-\tau_{1}, f\right)\right|^{2}+\left(1-\rho_{I_{\mathrm{UL}}}^{2}(t)\right) 2 \sigma^{2}+\sigma_{e}^{2}\right\} \leq \phi, \\
& P_{s_{\mathrm{DL}}}\left\{\rho_{I_{\mathrm{DL}}}^{2}(t)\left|\widehat{H}_{I_{\mathrm{DL}}}\left(-\tau_{2}, f\right)\right|^{2}+\left(1-\rho_{I_{\mathrm{DL}}}^{2}(t)\right) 2 \sigma^{2}+\sigma_{e}^{2}\right\} \leq \phi, \\
& \sum_{f=1}^{M} P_{s_{\mathrm{UL}}}(t, f) \leq \Phi, \quad-P_{s_{\mathrm{UL}}}(t, f) \leq 0, \\
& \sum_{f=1}^{M} P_{s_{\mathrm{DL}}}(t, f) \leq \Phi, \quad-P_{s_{\mathrm{DL}}}(t, f) \leq 0 .
\end{array}
$$

The solution is

$$
\begin{aligned}
& P_{s_{\mathrm{UL}}}(t, f)= \begin{cases}P_{D 1}, & \frac{B}{\mu \ln 2} \geq P_{D 1}+P_{D 2}, \\
\frac{B}{\mu \ln 2}-P_{D 2}, & P_{D 2}<\frac{B}{\mu \ln 2}<P_{D 1}+P_{D 2}, \\
0, & \frac{B}{\mu \ln 2} \leq P_{D 2},\end{cases} \\
& P_{s_{\mathrm{DL}}}(t, f)= \begin{cases}P_{D 3}, & \frac{B}{\mu \ln 2} \geq P_{D 3}+P_{D 4}, \\
\frac{B}{\mu \ln 2}-P_{D 4}, & P_{D 2}<\frac{B}{\mu \ln 2}<P_{D 3}+P_{D 4}, \\
0, & \frac{B}{\mu \ln 2} \leq P_{D 4},\end{cases}
\end{aligned}
$$

where $P_{D 1}=\phi /\left(\rho_{I_{\mathrm{UL}}}^{2}(t)\left|\widehat{H}_{I_{\mathrm{UL}}}\left(-\tau_{1}, f\right)\right|^{2}+\left(1-\rho_{I_{\mathrm{UL}}}^{2}(t)\right) 2 \sigma^{2}+\sigma_{e}^{2}\right)$, $P_{D 2}=\left(N_{0}+I_{p}\right) /\left(\rho_{I_{\mathrm{UL}}}^{2}(t)\left|\widehat{H}_{s_{\mathrm{UL}}}\left(-\tau_{s_{1}}, f\right)\right|^{2}+\left(1-\rho_{s_{\mathrm{UL}}}^{2}(t)\right) 2 \sigma^{2}+\sigma_{e}^{2}\right)$, $P_{D 3}=\phi /\left(\rho_{I_{\mathrm{DL}}}^{2}(t)\left|\widehat{H}_{I_{\mathrm{DL}}}\left(-\tau_{2}, f\right)\right|^{2}+\left(1-\rho_{I_{\mathrm{DL}}}^{2}(t)\right) 2 \sigma^{2}+\sigma_{e}^{2}\right), P_{D 4}=$ $\left(N_{0}+I_{p}\right) /\left(\rho_{I_{\mathrm{DL}}}^{2}(t)\left|\widehat{H}_{s_{\mathrm{DL}}}\left(-\tau_{s_{2}}, f\right)\right|^{2}+\left(1-\rho_{s_{\mathrm{DL}}}^{2}(t)\right) 2 \sigma^{2}+\sigma_{e}^{2}\right)$, and $\mu$ is the Lagrange multiplier.

\section{Simulation Results}

In this section, we compare the interference control performance using different interference constraints. The primary

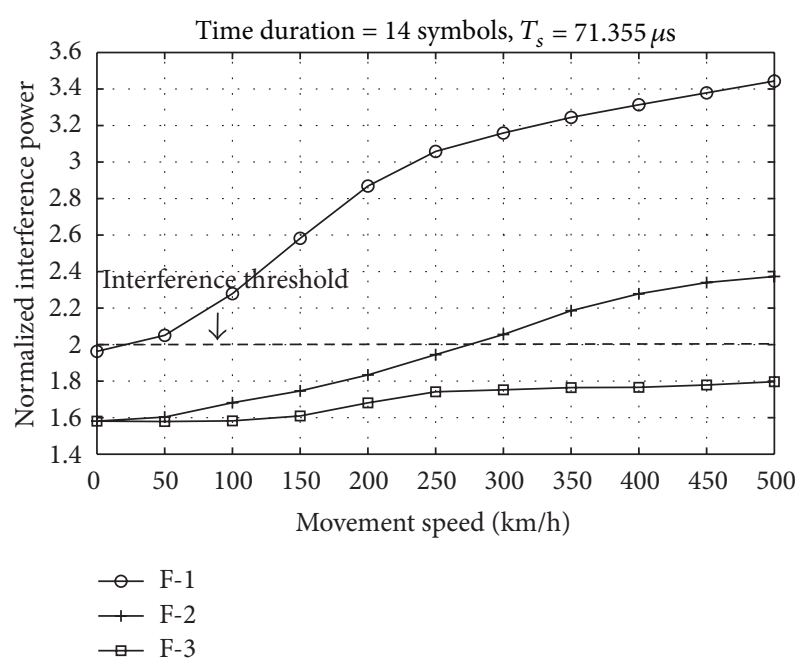

FIGURE 5: The mean interference power with different movement speeds.

system and secondary system have 512 subcarriers which are divided into 16 subbands. The carrier frequency is $2 \mathrm{GHz}$, and the subcarrier spacing is $15 \mathrm{kHz}$. Each OFDM symbol of duration is $71.355 \mu \mathrm{s}$. We set the time delay $\tau_{s_{1}}=0, \tau_{s_{2}}=0$, $\tau_{1}=0$, and $\tau_{2}=0$.

The time-varying fading channel has 16 uncorrelated Rayleigh fading taps; each tap has $3 \mathrm{~dB}$ decay factor. The timevarying fading channel is described by Clark-Jakes' model. The noise power is $N_{0}=1$. We set $\sigma^{2}=0.5$, and the maximum transmission power with secondary system is $\Phi=64$. The predefined interference threshold is $\phi=2, I_{p}=10$. The variance of channel estimation error is $\sigma_{e}^{2}=0.1$.

We compare the performance of the different IC schemes in mobile environment. The mean interference power is shown in Figure 5. Since the traditional IC (F-1) is designed based on the assumption of quasistatic channel, the interference constraints are only validated in motionless or slow movement condition (less than $30 \mathrm{~km} / \mathrm{h}$ ). As the increased movement speed, the interference power will exceed the interference threshold rapidly. The short frame structure (F-2) can guarantee the interference power under the interference threshold under $250 \mathrm{~km} / \mathrm{h}$, but the performance of IC becomes worse rapidly when speed approaches $500 \mathrm{~km} / \mathrm{h}$. The (F-3) curve shows the IC performance of the mean interference power constraint in time-varying fading channel; the secondary system limits their transmission power in uplink and downlink. The higher movement speed will lead to worse IC performance. Comparing the three different constraints, the secondary system should adopt the mean interference constraint in high mobility environment to satisfy the QoS demand primary system.

The interference power changes from symbol to symbol in the time-varying fading channel at $300 \mathrm{~km} / \mathrm{h}$ as shown in Figure 6. The channel correlationship can be described by Clark-Jakes' model which follows the 0th order Bessel function $J_{0}$; the channel correlation coefficient $\rho$ is a variable of time which has been shown in Figure 3. Since the proposed 


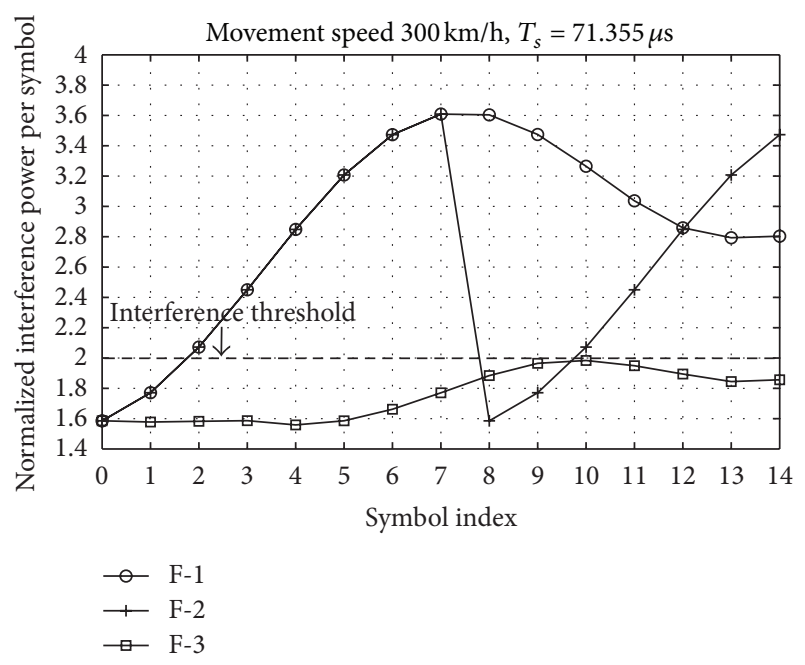

FIGURE 6: The interference power per symbol at primary receiver.

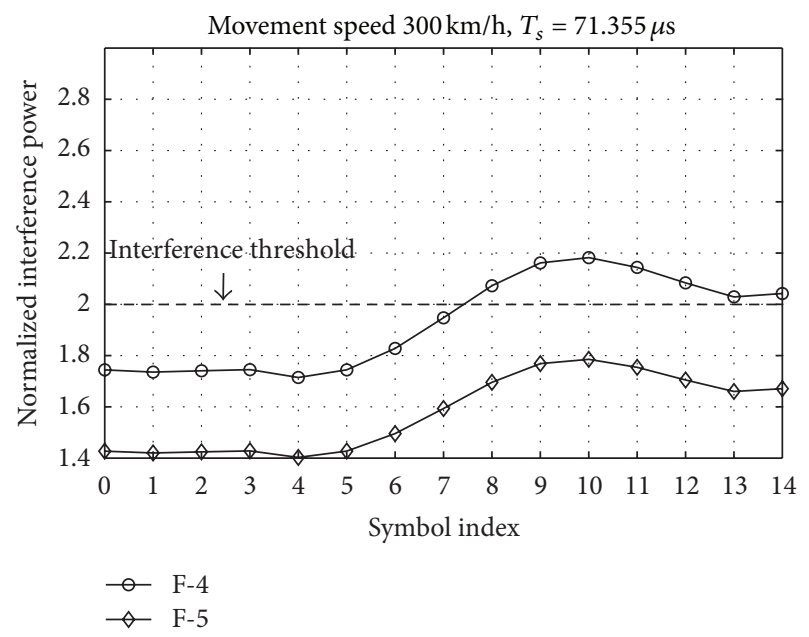

Figure 7: Mean interference power at the primary receiver.

interference constraints are impacted by $\rho^{2}$, the performance of the interference constraint is related to the change of the channel correlation coefficient. The value of $\rho^{2}$ will decrease from 1 to 0 in the first 9 symbols and then increase from the 10th symbol. Therefore, as shown in Figure 6, the trend of the interference power has the convex and wavy features. The traditional IC constraint with quasistatic assumption (F-1) cannot reflect the variation of the timevarying channel. The interference constraint cannot ensure the interference power under threshold after 2 symbols, and the maximum interference power is above 3.6. As the curve (F-2) is shown, the interference power seriously decreases at the 8th symbol which means that the short frame structure can lead to more frequently IC than standard frame length. The IC performance with short frame structure is better than traditional way to constrain the interference power. However, the communication overhead of short frame IC is twice as much as standard frame structure, which spends more time on channel estimation, thus wasting the opportunity of data transmission. When the channel state changes fast, it is impossible to control the interference by shorting the frame length unlimitedly. To cope with the IC problem in high mobility environment, the IC with mean interference constraint can be seen as a more effective way to control the interference power. The performance of IC with mean interference power constraint (F-3) shows that it perfectly limits the interference power under the interference threshold.

Considering the influence of the channel estimation error, the performance of different IC schemes at $300 \mathrm{~km} / \mathrm{h}$ is shown in Figure 7. Because of the IC relies on the accuracy CSI, the influence of estimation error with CSI cannot be ignored. The actual interference power may exceed the interference threshold with the error-existed CSI. It is observed that the IC scheme which considers the mean interference constraint with imperfect channel estimation (F-4) may become invalid after several symbols. From the results shown in Figure 7, it can be found that constraining the interference power with considering the maximum radius of error bound is a conservative IC scheme. The interference power can be limited under the interference threshold within error-existed CSI. The results show that our proposed scheme is robust when the channel estimation error exists.

\section{Conclusion}

In underlay CRNs, limiting the interference power to the primary system is an important topic for the secondary system optimization. The existing IC schemes were based on the quasistatic channel assumption, but the variation of channel in a frame duration cannot be neglected in the high mobility environment. In this paper, we have developed the IC schemes in mobile environment considering the influence of the time-varying fading channel and the channel estimation error. The proposed approaches can limit the interference power under the predefined interference threshold in the mobile environment. Future directions include the IC schemes with the assumption of nonsynchronous transmission process between the primary system and the secondary system and the multiple antennas IC schemes in mobile environment.

\section{Acknowledgments}

This work is supported by the Fundamental Research Funds for the Central Universities under Grant no. 2012YJS017, Key Project of State Key Laboratory of Rail Traffic Control and Safety under Grant no. RCS2012ZZ004, Key Grant Project of Chinese Ministry of Education no. 313006, and Program for New Century Excellent Talents in University under Grant NCET-09-0206.

\section{References}

[1] FCC, Spectrum Policy Task Force, Proceedings of the Federal Communications Commission, Washington, DC, USA, 2002.

[2] J. Mitola, "Cognitive radio architecture evolution," Proceedings of the IEEE, vol. 97, no. 4, pp. 626-641, 2009. 
[3] O. Simeone, Y. Bar-Ness, and U. Spagnolini, "Stable throughput of cognitive radios with and without relaying capability," IEEE Transactions on Communications, vol. 55, no. 12, pp. 2351-2360, 2007.

[4] J. Mietzner, L. Lampe, and R. Schober, "Distributed transmit power allocation for multihop cognitive-radio systems," IEEE Transactions on Wireless Communications, vol. 8, no. 10, pp. 5187-5201, 2009.

[5] M. Gastpar, "On capacity under receive and spatial spectrumsharing constraints," IEEE Transactions on Information Theory, vol. 53, no. 2, pp. 471-487, 2007.

[6] A. Goldsmith, S. A. Jafar, I. Maric, and S. Srinivasa, "Breaking spectrum gridlock with cognitive radios: an information theoretic perspective," Proceedings of the IEEE, vol. 97, no. 5, pp. 894-914, 2009.

[7] H. Yao, Z. Zhou, H. Liu, and L. Zhang, "Optimal power allocation in joint spectrum underlay and overlay cognitive radio networks," in Proceedings of the 4th International Conference on Cognitive Radio Oriented Wireless Networks and Communications (CROWNCOM '09), pp. 1-5, June 2009.

[8] L. Le and E. Hossain, "Resource allocation for spectrum underlay in cognitive radio networks," IEEE Transactions on Wireless Communications, vol. 7, no. 12, pp. 5306-5315, 2008.

[9] M. Z. Win, P. C. Pinto, and L. A. Shepp, "A mathematical theory of network interference and its applications," Proceedings of the IEEE, vol. 97, no. 2, pp. 205-230, 2009.

[10] A. Rabbachin, T. Q. S. Quek, H. Shin, and M. Z. Win, "Cognitive network interference," IEEE Journal on Selected Areas in Communications, vol. 29, no. 2, pp. 480-493, 2011.

[11] K. Guan, Z. Zhong, and B. Ai, "Assessment of LTE-R using high speed railway channel model," in Proceedings of the $3 \mathrm{rd}$ IEEE International Conference on Communications and Mobile Computing (CMC '11), pp. 461-464, April 2011.

[12] W. Qiu, B. Xie, H. Minn, and C. Chong, "Interferencecontrolled transmission schemes for cognitive radio in frequency-selective time-varying fading channels," IEEE Transactions on Wireless Communications, vol. 11, no. 1, pp. 142-153, 2012.

[13] M. Patzold, Mobile Fading Channels, John Wiley \& Sons, New York, NY, USA, 2002.

[14] M. B. Shenouda and T. N. Davidson, "Convex conic formulations of robust downlink precoder designs with quality of service constraints," IEEE Journal on Selected Topics in Signal Processing, vol. 1, no. 4, pp. 714-724, 2007. 


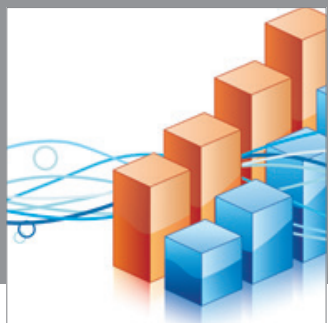

Advances in

Operations Research

mansans

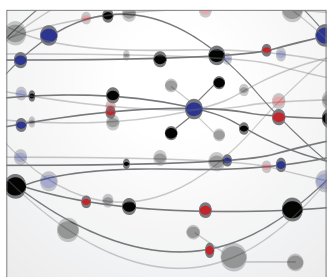

The Scientific World Journal
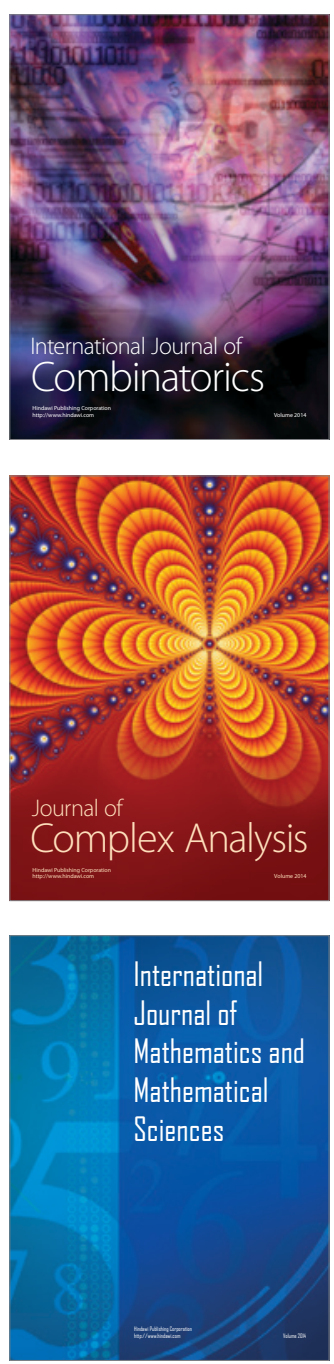
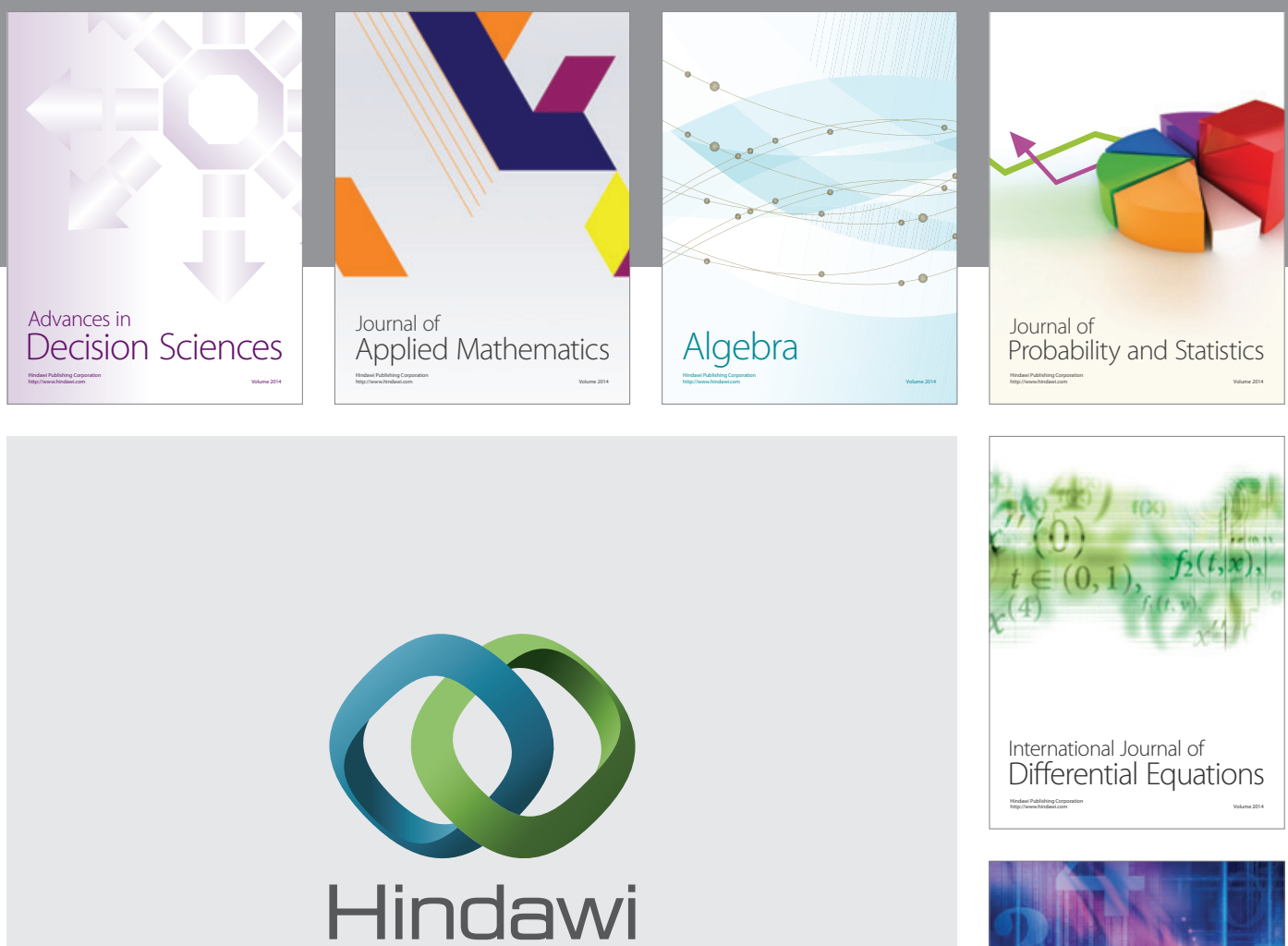

Submit your manuscripts at http://www.hindawi.com
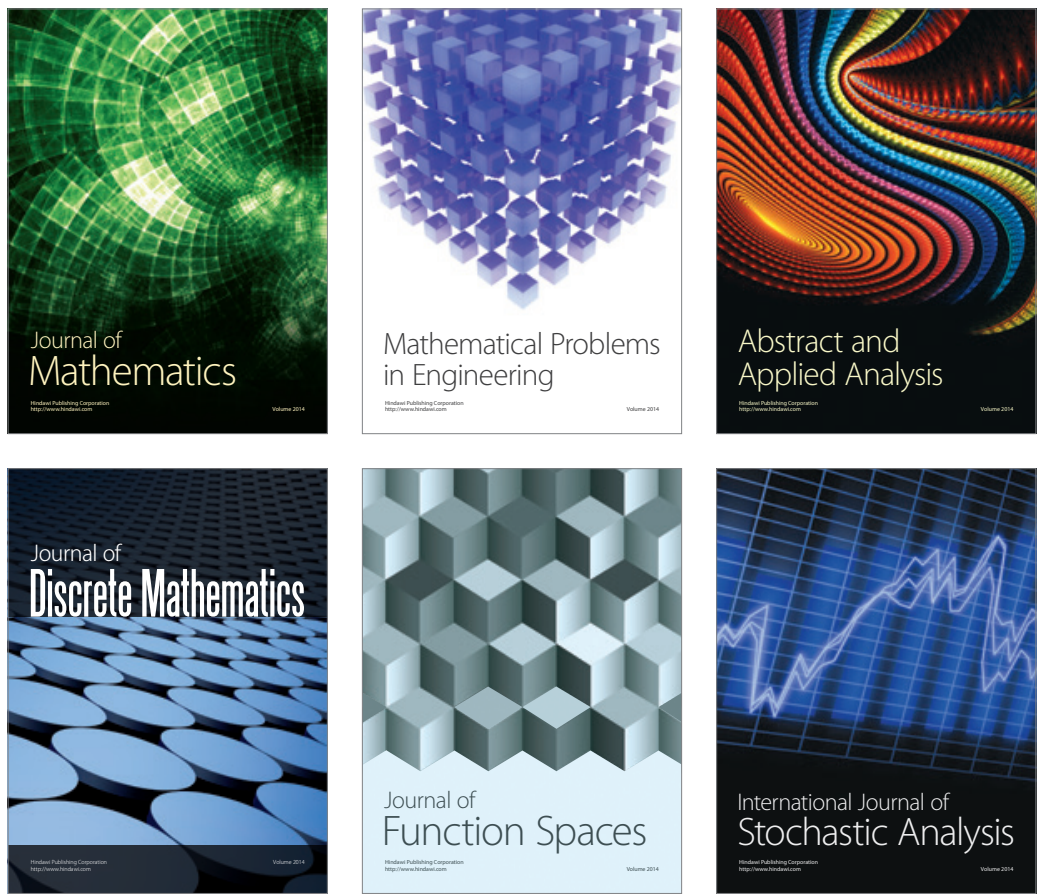

Journal of

Function Spaces

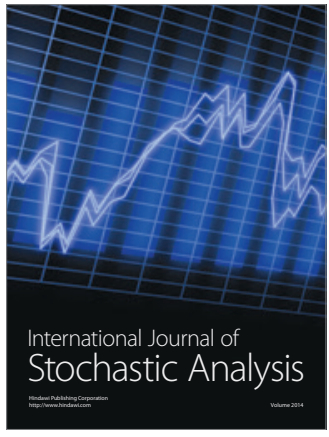

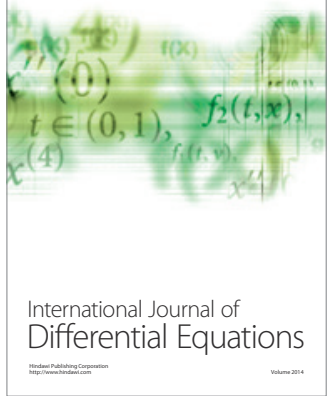
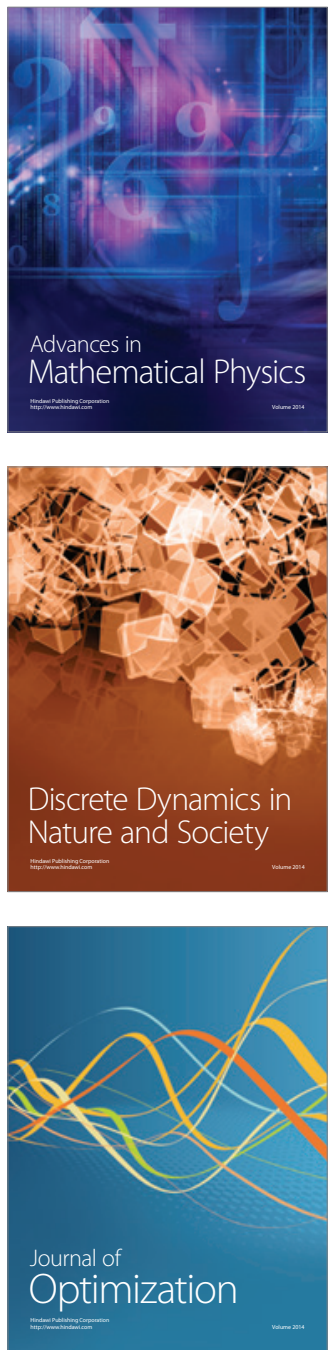